$\mathbb{T}$ periodica polytechnica

Mechanical Engineering

$52 / 1(2008) 9$

doi: 10.3311/pp.me.2008-1.02

web: http://www.pp.bme.hu/me

(C) Periodica Polytechnica 2008

RESEARCH ARTICLE

\section{Analysis of colour moiré phenomenon in colour space}

\author{
Daria Pavelyeva / Klára Wenzel
}

Received 2008-01-18

\begin{abstract}
Moiré topographic technique is widely used for threedimensional surface reconstruction. One of the main problems is determination of the absolute order of a fringe for finding the depth difference between two points in moiré patterns, $i$. e. convex and concave surfaces distinction. Most solutions of the problem are very complicated; also they usually require several moiré images. Application of colour-encoded gratings in projection moiré method allows solving the problem simply and quickly. The authors analyze the information that contains in colour moiré fringes and estimate its effectiveness. With this purpose device independent simulations for colour formation of one moiré fringe in colour space by different conditions were made.
\end{abstract}

\section{Keywords}

surface shape identification - moiré pattern · uniform colour space.

\section{Acknowledgement}

The authors would like to express their sincere thanks to Antal A. for help during the experiments and many valuable consultations.

\section{Daria Pavelyeva}

Department of Mechatronics, Optics and Engineering Informatics, BME, H1111 Budapest, Múegyetem rkp. 5., Hungary

e-mail: daria_pavelyeva@mogi.bme.hu

\section{Klára Wenzel}

Department of Mechatronics, Optics and Engineering Informatics, BME, H1111 Budapest, Múegyetem rkp. 5., Hungary

e-mail: awenzel@mogi.bme.hu

\section{Introduction}

Moiré phenomenon arises wherever a repetitive structure is overlaid with another structure and the line elements are nearly superimposed. Moiré methods are termed such due to their use of the moiré effect to make measurements. Within moiré measurement methods, many techniques exist. Out-of-plane measurement methods are shadow [1] and projection moiré method [2]. Moiré topographic technique is a well known and powerful tool for three-dimensional surface reconstruction and analysis of the real objects [3].

One of the main problems by moiré topographic methods is the arising ambiguities of depth between two points in the moiré patterns, or so called "hill and valley" problem. The fringe carrier technique [4] and temporal phase-shift technique [5] are common used methods for solving this problem. But phase shifting techniques require several steps of precise phase shifting, limiting its application. The fringe carrier method is restricted by its carrier fringe frequency. Several methods with application of colour to moiré methods have been already proposed [6], [7], [8]. However the main disadvantage of the earlier presented methods is complication of mechanical and/or analyzing process. Recently the new simple method that solves the problem of ambiguities from one moiré pattern by application of colour was presented [9]. The authors suggested using two special colour-encoded fringe patterns in spite of common Ronchi gratings for projection moiré method. But the question dedicated to selection of colours spectral characteristics, used in the method, was not considered. In this paper we present analysis of colour moiré phenomenon in colour space that allows finding appropriate colour stimuli for surfaces with different spectral characteristics.

In colour printing technology moiré patterns display an unexpected new frequency and orientation [10]. Many algorithms were worked out for minimization of unwanted moiré effect. From our point of view colour moiré phenomenon gives us extra information that we can use for solution of "hill and valley" problem in moiré patterns. 


\section{Theory}

Moiré projection method entails projecting a fringe pattern or grating on the test object and viewing it through analyzer grating from a different direction (Fig. 1).

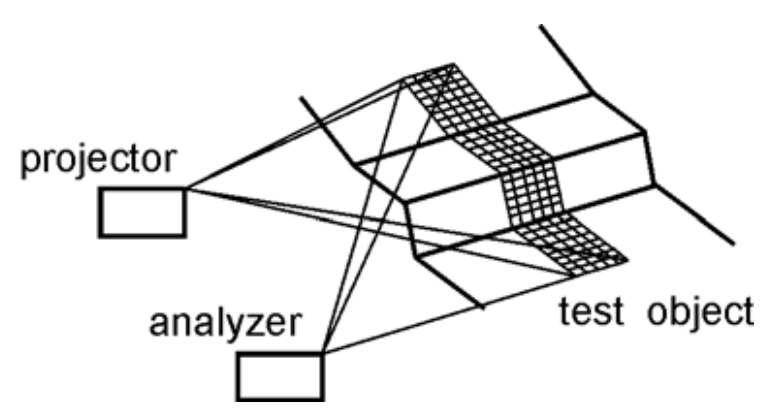

Fig. 1. Projection moiré method

In colour moiré projection method authors used two uniform colour-encoded gratings for analyzer and projection fringe patterns. The gratings have opaque and transparent bars of the equal width, where each opaque bar consists of two rulings with different spectral transmittances. Fig. 2 a presents the classical moiré effect obtained by two black\&white gratings superimposition.

Computer generated colour moiré patterns generated by two colour gratings with the same and opposite order of bars are shown accordingly in Fig. 2p and Fig. 2k.

The straight lines cutting through the moiré fringes in the pictures show the transformation of colour in one period of moiré fringe (Fig. 2a $, \mathrm{b}, \mathrm{c}$ ).

The colour phenomenon shown in Fig. $2 \mathrm{p}$ is ,effective”, since it was proved earlier that changing colour in moiré fringe allows distinguishing convex and concave surfaces in moiré patterns [9] (Fig. 3). Accordingly ,ineffective” case will be colour moiré phenomenon with uniform colour over one period of moiré fringe as it gives no extra information for overcoming the problem of ambiguities in moiré images (Fig.2p).

\subsection{Analysis of colour projection moiré phenomenon in colour space}

As it was already mentioned the goal of our research to analyze the colour moiré in colour space. It will help to find appropriate colour stimuli of bars in fringe gratings for measuring objects with different spectral characteristics. During our work we made device independent model of colour projection moiré phenomenon formation, i.e. as analyzer no artificial device was applied but human eye.

Thus, using relative spectral power distribution of the illuminant, spectral reflectance factor of the test object colour and spectral transmittance of the colour bar in the films we calculated the relative colour stimulus functions of the colours in moiré fringe

$$
\phi(\lambda)=S(\lambda) \tau_{p}(\lambda) R(\lambda) \tau_{a}(\lambda)
$$

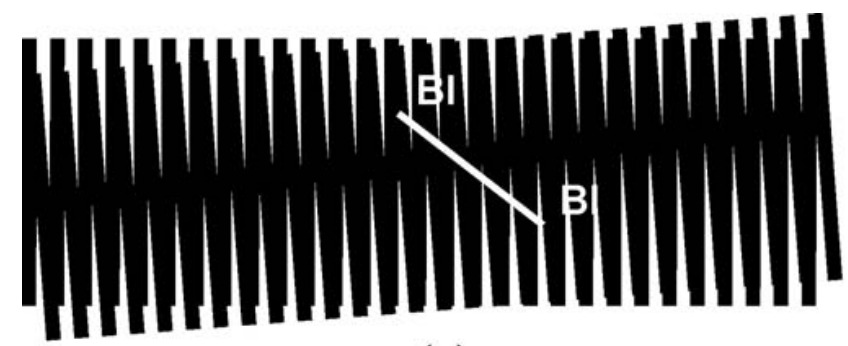

(a)

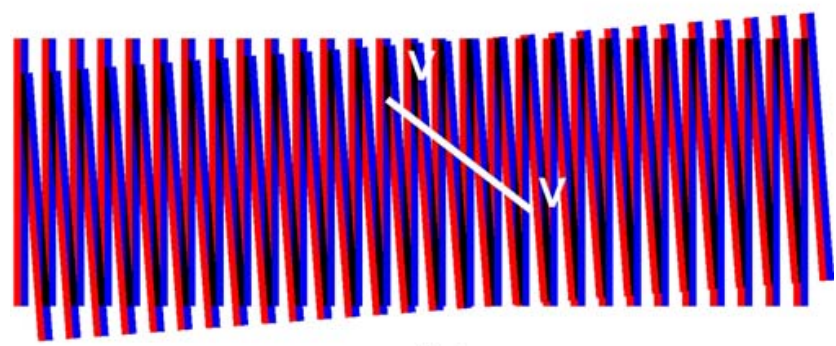

(b)

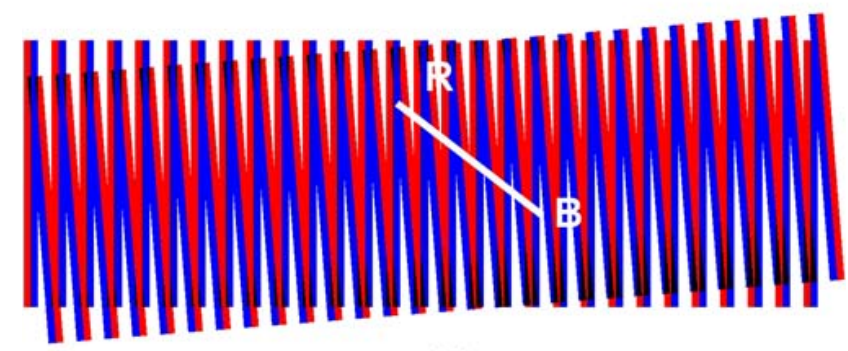

(c)

Fig. 2. Classical (a), „effective” (b) and ,ineffective” (c) cases of colour moiré phenomenon formation. The straight lines cutting through the moiré fringes in the pictures show the transformation of colour in one period of moiré fringe Bl - black, V - violet, B- blue, R - red

where $\phi_{\lambda}(\lambda)$ - relative colour stimulus function of colour by looking through analyzer grating, $S(\lambda)$ - relative spectral power distribution of the illuminant, $\tau_{p}(\lambda)$ - spectral transmittance of the colour bar in the film of the projection grating, $\tau_{a}(\lambda)$ - spectral transmittance of the colour bar in the film of the analyzer grating, $\left(\tau_{a}(\lambda)\right.$ and $\tau_{p}(\lambda)$ contain the information of the base and colour emulsion), $R(\lambda)$ - spectral reflectance factor of the test object colour.

As long as the both gratings contain two colour - encoded opaque and transparent bars. It means that we have six colours, which appear in one period of moiré fringe. For definition of participate colours proportions the graphic model of one period of moiré fringe in relative values was made (Fig. 2p,c). The CIE tristimulus values of a colour stimulus can be found with formulas:

$$
\begin{aligned}
X & =k \sum_{\lambda} \phi_{\lambda}(\lambda) \bar{x} \Delta \lambda \\
Y & =k \sum_{\lambda} \phi_{\lambda}(\lambda) \bar{y} \Delta \lambda \\
Z & =k \sum_{\lambda} \phi_{\lambda}(\lambda) \bar{z} \Delta \lambda
\end{aligned}
$$




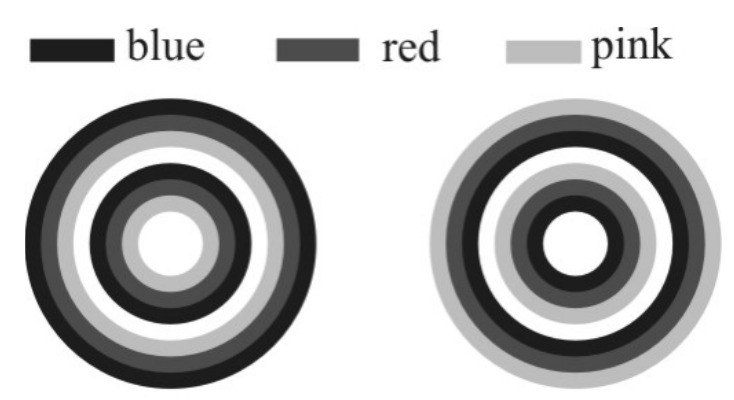

Fig. 3. Concave (a) and convex (b) surfaces by application of „effective” colour moiré phenomenon [9]

where $\mathrm{X}, \mathrm{Y}, \mathrm{Z}$ are the tristimulus values, $\bar{x}(\lambda), \bar{y}(\lambda), \bar{z}(\lambda)$ are colour-matching functions of a standard colorimetric observer, $\Delta \lambda$ is wavelength interval, $k$ is a normalizing constant [11]. The chromaticity coordinates $x, y, z$ are derived from the tristimulus values:

$$
\begin{aligned}
& x=\frac{X}{X+Y+Z} \\
& y=\frac{Y}{X+Y+Z} \\
& z=\frac{Z}{X+Y+Z}
\end{aligned}
$$

Now construct a graph of one period of colour moiré fringe in the CIE $x, y$ chromaticity diagram can be constructed. We will get two possible graphs modes. The first one conforms to „effective" case where the graph has the form different to line, i. e. colours in one period of moiré fringe are nonrepeatable (Fig. 4a). The second graph is named ,ineffective" one and has the form of line that means colours repeat in one period of moire fringe (Fig. 44).

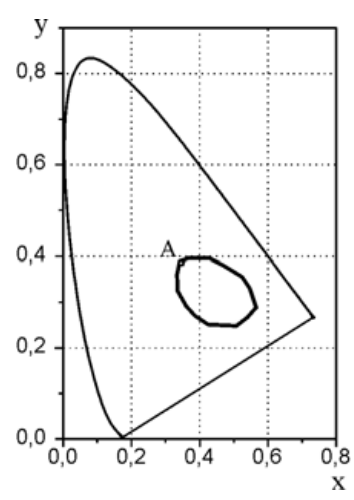

(a)

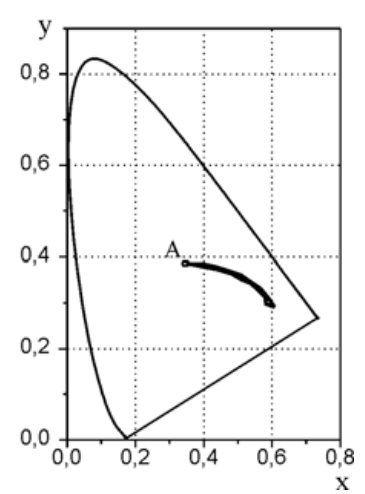

(b)
Fig. 4. „Effective” (a) and ,ineffective” (b) colour moiré phenomenon in the CIE $x, y$ chromaticity diagram.

Thus, by the form of graph in the CIE $x, y$ chromaticity diagram we can chose ,effective " cases among ,ineffective” ones. But if there are several ,effective” cases (Fig. 5), which of them will be the best or by other words has the highest contrast of changing colour in the fringe?

We suggest solving the problem by calculation of the colour difference between the two colours: the first colour represents

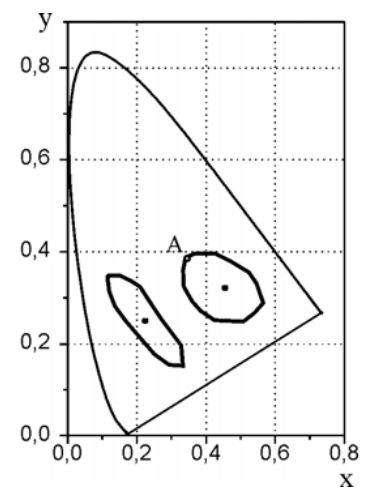

(a) (b)

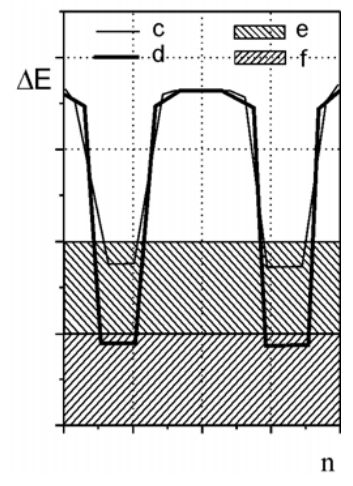

Fig. 5. Example of two „effective” colour moiré phenomena in the (a) CIE $\mathrm{x}, \mathrm{y}$ chromaticity diagram and (b) response diagram of colour difference $\Delta E_{u v}^{*}$ from disposition of colours lying on the line perpendicular to the rulings of the grating in one moiré fringe period; $c--$ less ,effective” and d-more ,effective” colour moiré effect, $f$ - zone of just-noticeable colour difference and $e$ - zone of just-acceptable colour difference.

the result of an additive mixture of colours composing one period of moiré fringe and the second colour - separate colour of one period of moiré fringe.

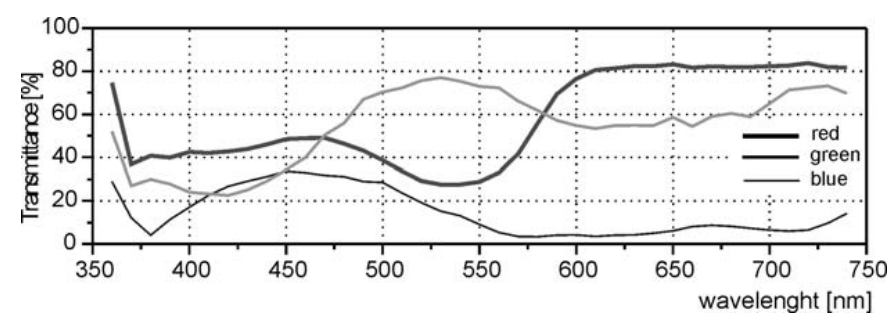

Fig. 6. Spectral transmittance curves of red, green and blue colours

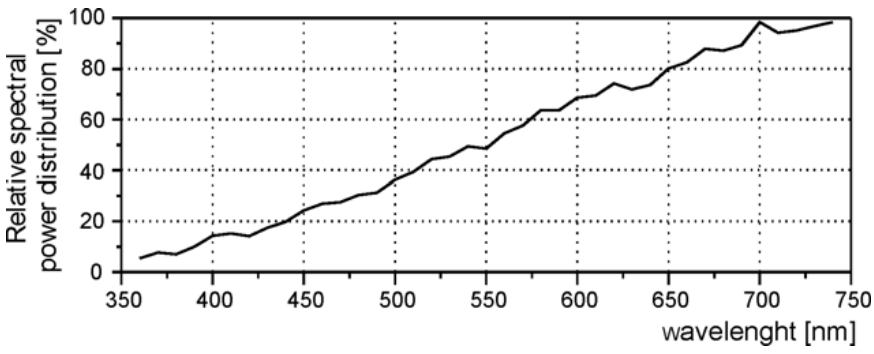

Fig. 7. Relative spectral power distribution of halogen lamp

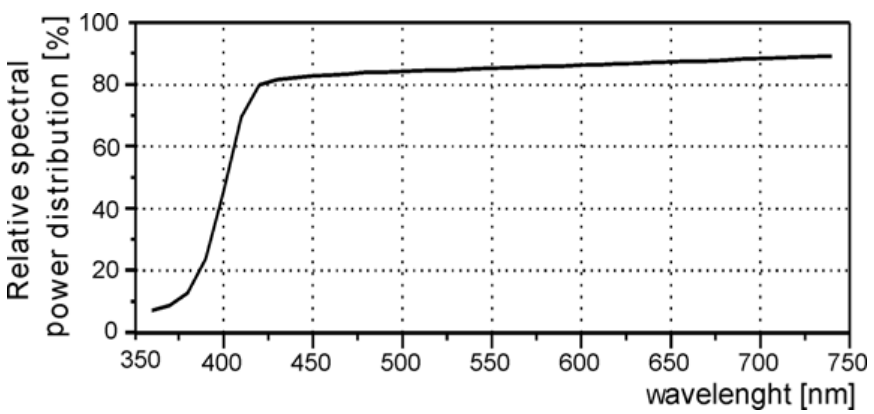

Fig. 8. Spectral reflection curve of the test surface

In the CIE $\mathrm{x}, \mathrm{y}$ chromaticity diagram the chromaticity point 
of two additive mixed colours is located on the line joining the chromaticity points of the two constituent colours. An additive mixture takes advantage of the limited resolving power of the eye. Thus, colour moiré phenomenon, composed of six colours, appears a mixture of that colours when viewed from such a distance that the individual colour stimuli can not be resolved. If two colours are specified of $Y_{1}, x_{1}, y_{1}$ and $Y_{2}, x_{2}, y_{2}$, the luminance and chromaticity of the additive mixture can be found by formulae:

$$
\begin{gathered}
Y=Y_{1}+Y_{2} \\
x=\frac{m_{1} x_{1}+m_{2} x_{2}}{m_{1}+m_{2}} \\
y=\frac{m_{1} y_{1}+m_{2} y_{2}}{m_{1}+m_{2}}
\end{gathered}
$$

where $m_{1}=\frac{Y_{1}}{y_{1}}$ and $m_{2}=\frac{Y_{2}}{y_{2}}$. The equations for $x$ and $y$ are familiar to the center of gravity of two masses $m_{1}$ and $m_{2}$ located at $x_{1} y_{1}$, and $x_{2} y_{2}$, respectively.

For calculation of colour difference we chose the CIE 1976 $\left(\mathrm{L}^{*} \mathrm{u}^{*} \mathrm{v}^{*}\right)$ three-dimensional, approximately uniform colour space. The CIE $1976\left(\mathrm{~L}^{*} \mathrm{u}^{*} \mathrm{v}^{*}\right.$ ) is widely used in calculation of small colours or colour differences, especially with additive colours. This colour space comprises the $\left(u^{\prime}, v^{\prime}\right)$ colour diagram relying on projective transformation of the $(x, y)$ chromaticity diagram. In the CIE $1976\left(\mathrm{~L}^{*} \mathrm{u}^{*} \mathrm{v}^{*}\right)$, products of additive colour mixing fall on a straight line, just as in the $(x, y)$ chromaticity diagram. The difference between two colour stimuli can be calculated from formulae:

$$
\begin{gathered}
\Delta E_{u v}^{*}=\left[\left(\Delta L^{*}\right)^{2}+\left(\Delta u^{*}\right)^{2}+\left(\Delta v^{*}\right)^{2}\right]^{1 / 2} \\
L^{*}=116\left(\frac{Y}{Y_{n}}\right)-16 \\
u^{*}=13 L^{*}\left(u^{\prime}-u_{n}^{\prime}\right) ; v^{*}=13 L^{*}\left(v^{\prime}-v_{n}^{\prime}\right) \\
u^{\prime}=\frac{4 x}{-2 x+12 y+3} ; v^{\prime}=\frac{9 y}{-2 x+12 y+3} \\
f\left(\frac{Y}{Y_{n}}\right)=\left(\frac{Y}{Y_{n}}\right)^{\frac{1}{3}}, \text { if } \frac{Y}{Y_{n}}>0,00856 \text { and } \\
f\left(\frac{Y}{Y_{n}}\right)=7,787 \frac{Y}{Y_{0}}+\frac{16}{116}, \text { if } \frac{Y}{Y_{n}} \leq 0,00856
\end{gathered}
$$

where $\Delta E_{u v}^{*}$ is the difference between two colour stimuli, $\mathrm{L}^{*}$ component defines the luminancy, $\mathrm{u}^{*}$ and $\mathrm{v}^{*}$ define chrominancy, $\mathrm{Y}, u^{\prime}, v^{\prime}$ describe the colour stimulus and $\mathrm{Y}_{n}, u_{n}^{\prime}, v_{n}^{\prime}$ describe a specified white object colour stimulus.

Then, by construction the response diagram of colour difference $\Delta E_{u v}^{*}$ from disposition of colours lying on the line perpendicular to the rulings of the grating in one moiré fringe period the best case will be that one, where the minimum difference between two colour stimuli over a period of moiré fringe is the biggest (Fig. 5p).

\section{Experiments}

In experimental part we worked with proposed device independent model illustrated earlier. Two transparent films with segments of red, green and blue primary colour were made. The colours on the film were produced by laser (Osé 700) printer. The spectral transmittance curves of the colours were obtained by spectrophotometer (AvaSpec-1024 Fiber Optic Spectrometer) and presented in Fig. 6

A halogen lamp was used as illuminant, its relative spectral power distribution was observed by CCD-based Compact Array Spectrometer (CAS 140B) (Fig.7).

Test surface was deformed in such a way, that some kind of hill and valley appeared on it. Spectral reflection curve of it was measured by spectrophotometer (Konica - Minolta CM-3600D) and presented in Fig. 8 .

While all multipliers from the formula (1) are know and we can calculate six relative colour stimulus functions in the analyzer for each case of two colour- encoded gratings combination. Then sequentially applying formulae (2) - (6) we get the chromaticity coordinates $x, y$ of the colours and construct the graphs of one period of moiré fringe in the CIE $x, y$ chromaticity diagram. We chose the cases of ,effective” colour moiré phenomenon by the form of the graphs in the CIE $x, y$ chromaticity diagram - by our initial data these are cases, when order of colour bars in gratings is the opposite, i. e. BR-RB, BG-GB and GR-RG (Fig. 9a, b).

In Fig. 10a, b cases of ,ineffective" colour moiré phenomenon are shown, that conform the cases with application of colour bars of the grating in uniform order, i. e. BR-BR, BG-BG and GRGR.

Then making calculations by formulae (7)-(12), we find the colour difference between two colour stimuli, i. e. we can find the best variant of colours bars for the gratings.

Fig. 11 a, b presents the response diagrams of colour difference $\Delta E_{u v}^{*}$ from disposition of colours lying on the line perpendicular to the rulings of the grating in one moiré fringe period for „effective” and "ineffective” colour moiré phenomenon. From the Fig. 11 a graphs it is seen that the most „effective" colour moiré phenomenon arises when the combination of red and blue stripes is used in different sequence by superimposition of projecting and analyzer gratings. The diagrams presented in Fig. 11p concern to the ,effective" cases of the colour moiré effect appearance, as they contain such parts where the colour difference is too small to be observable by the human eye, according to science literature [12].

Fig. 12 a shows colour moiré image of the measuring object by application of gratings with opposite order of colour rulings (red and blue bars were used) in projection method. Line profile graphics of colour moiré fringes in moiré pattern are presented in Fig. 12 p. They illustrate the red green blue components of the data that was obtained by sampling along a straight line cutting through the moir fringes. DAC, digital-to-analog converter, 

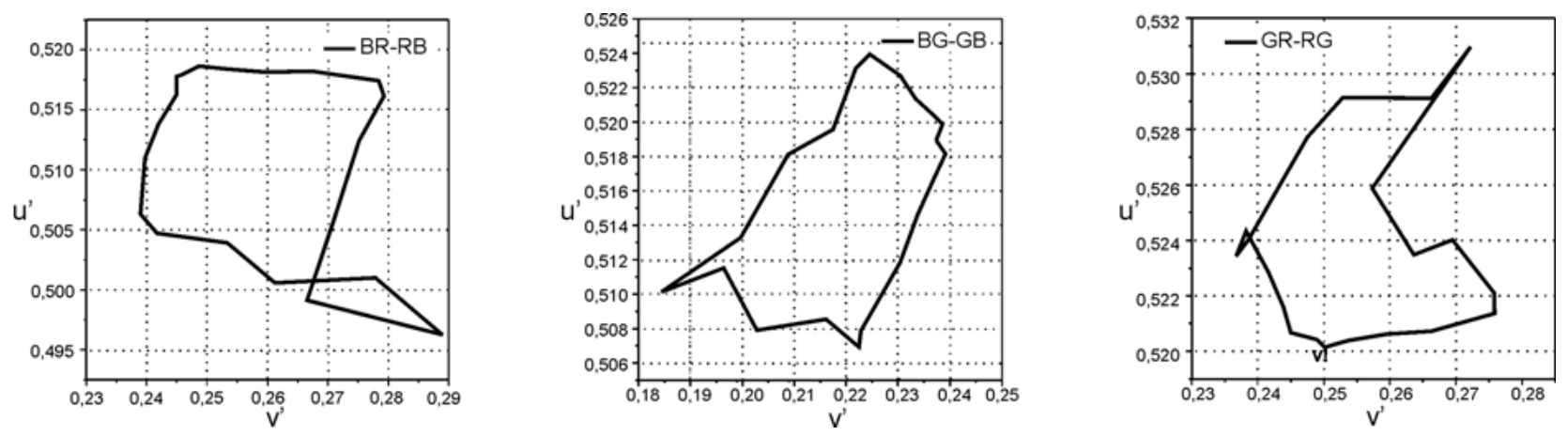

Fig. 9. „Effective” colour moiré phenomenon for gratings with opposite order of colour rulings in the CIE $x$, $y$ chromaticity diagram
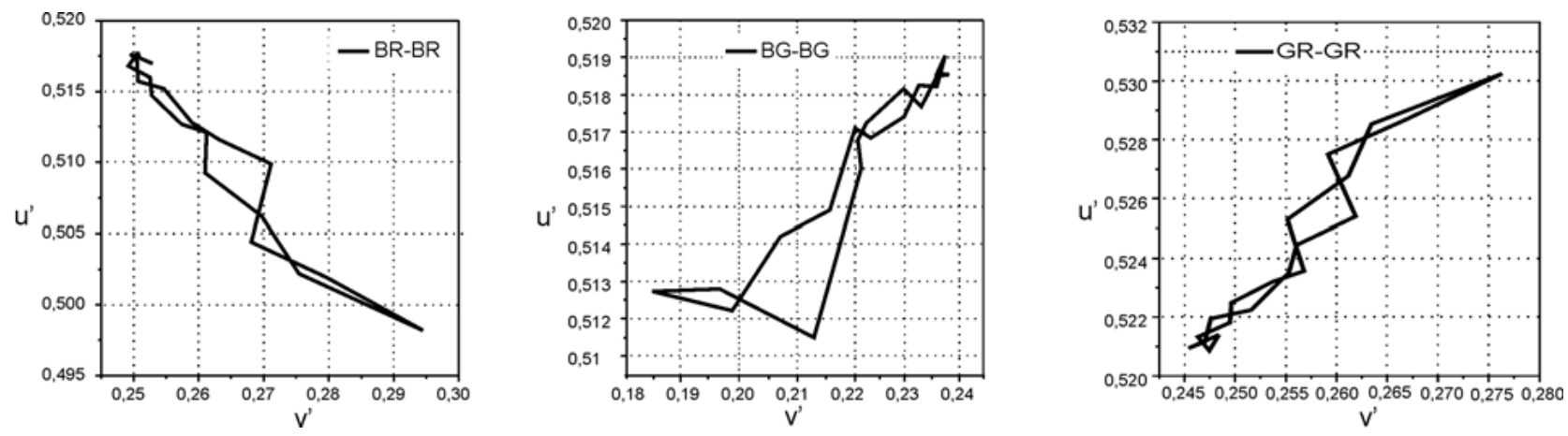

Fig. 10. „Ineffective” colour moiré phenomenon for gratings with uniform order of colour bars in the CIE $x, y$ chromaticity diagram

which is the measurement unit of the colour coordinates, that can take any integer value between 0 and 255 .

\section{Conclusions}

By device independent simulation and analyzing of the colour moiré phenomenon and colours sequence representation in colour spaces we made an explanation for experimental proved fact, that sequence of colour stripes in projection and analyzing gratings specifies the effectiveness of colour moiré phenomenon, i. e. its usability for distinction of convex and concave surfaces. During the work notions of ,effective” and ,ineffective" colour moiré phenomenon were put in, as long as by application of projection moiré method not any colour moiré phenomenon can be useful for solving the hill and valley problem. „Effective" colour moiré phenomenon gives extra information comparing to the classical black-white moiré effect about order of fringes in moiré patterns. Also we showed how to select the most „effective” phenomenon in case of several „effective” cases of colour moiré effect. By proposed algorithm one can find optimal spectral characteristics of colours in gratings and in such a way get quality colour moiré images.

\section{References}

1 Takasaki H, Moiré Topography, Applied Optics 9 (1970), no. 6, 1467-1472, DOI 10.1364/AO.9.001467.

2 Tay C, Thakur M, Quan C, Grating projection system for surface contour measurement, Applied Optics 44 (2005), no. 8, 1393-1400, DOI 10.1364/AO.44.001393.

3 Antal Á, Ölveczky B, Spectral Analysis of Moiré Images, Per. Pol. Mech. Eng. 41 (1997), no. 2, 85-93.
4 Plotknowski PD, Improved Fringe Carrier Technique for Unambiguous Determinations of Holographically Recorded Displacements, Optical Engineering 24 (1985), 754-764.

5 Sirivasan V, Automated Phase-measuring Profilometry of 3-D Diffuse Objects, Applied Optics 23 (1984), 3105-3114.

6 Chang-Hua Hu-Yu-Wen Qin, Digital Color Encoding and its Application to the Moiré Technique, Applied Optics 35 (1997), 3682-3685.

7 Harding KG, Coletta MP, Vandommelen CH, Color Encoded Moiré Contouring, Optics, Illumination, and Image Sensing for Machine Vision III, Proc. SPIE 1005 (1988), 169-178.

8 Livnat A, Kafri O, Erez G, Hills and Valleys Analysis in Optical Mapping and Its Application to Moiré Contouring, Applies Optics 19 (1980), 33983400, DOI 10.1364/AO.19.003396.

9 Antal A, Paveleva D, Projection Method of Resolving Ambiguities by Determining the Order of Colors in Moiré Fringes, Applied Optics 44 (2005), no. 36, 7709-7713, DOI 10.1364/AO.44.007709.

10 Ville D, Philips W, Lemahieu I, Walle A, Suppression of Sampling Moiré in Color Printing by Spline-based Least-squares Prefiltering, Pattern Recognition Letters 24 (2003), 1787-1794.

11 Commission Internationale de l'Eclairage,Colorimetry, Publication CIE, 1986.

12 Routine Test Method for Paper and Board. Instrumental Measurement of Colour, Paper Technology and Industry (1977), 217-218. 


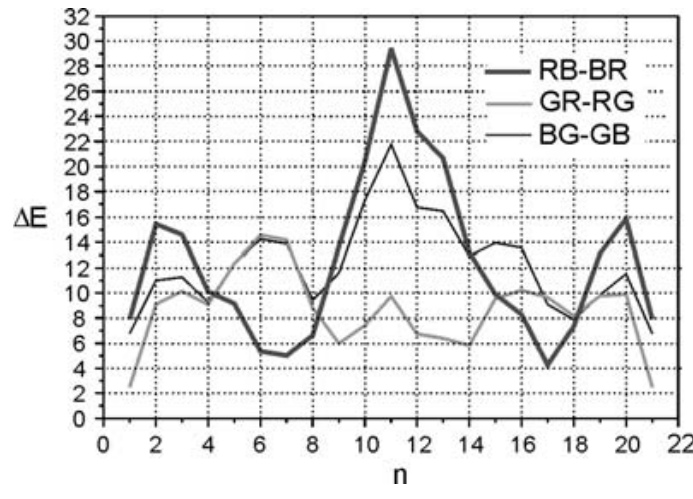

(a)

Fig. 11. Response diagrams of colour difference $\Delta E_{u v}^{*}$ from disposition of colours lying on the line perpendicular to the rulings of the grating in one moire

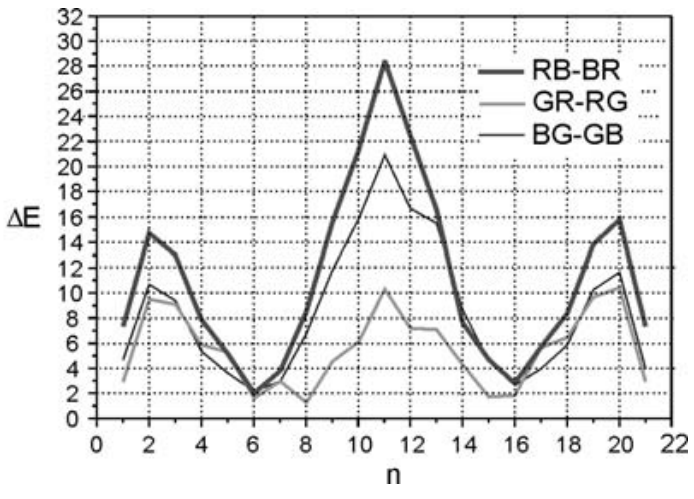

(b)

fringe period. Diagrams of „effective” (a) and „ineffective” (b) colour moiré fringes

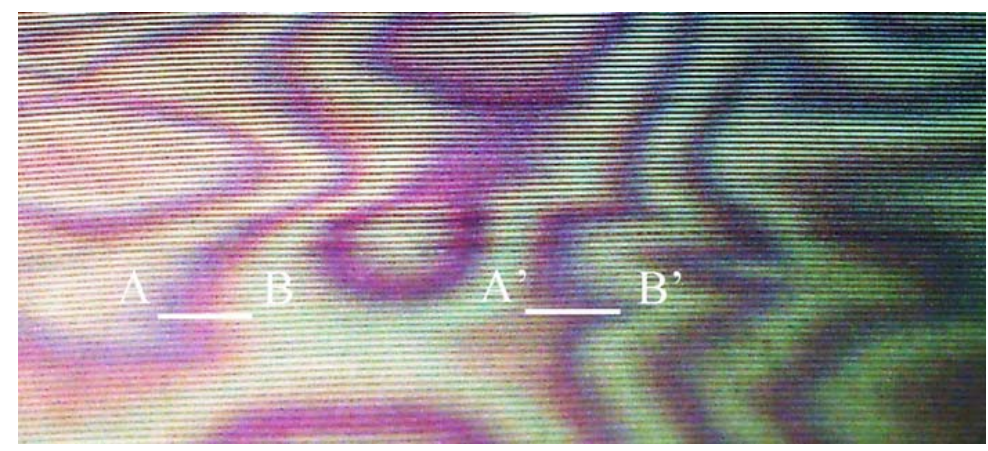

(a)
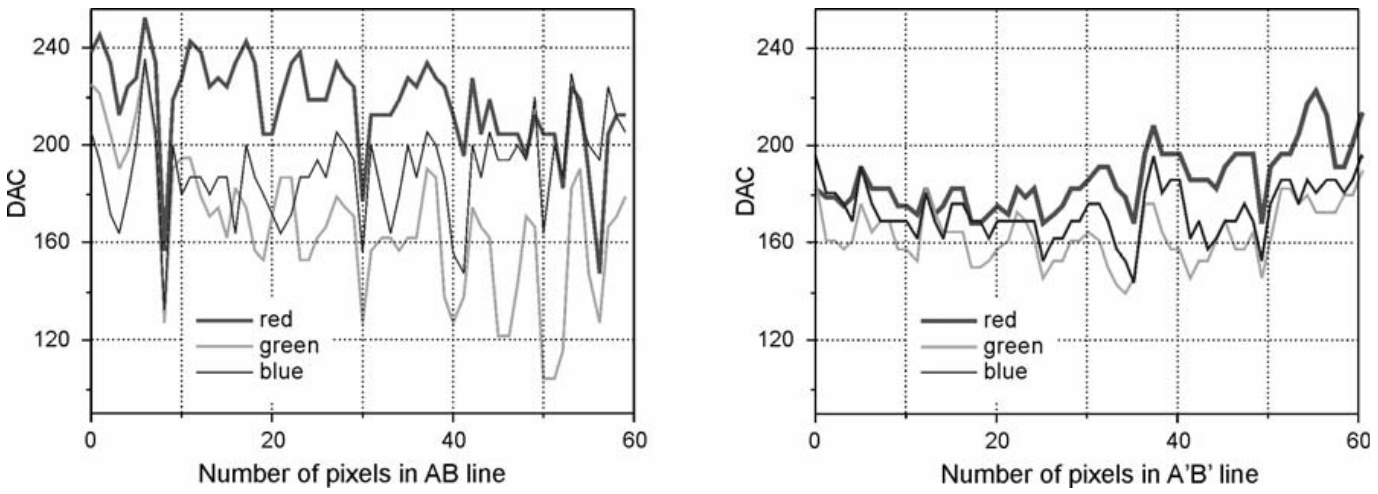

(b)

Fig. 12. Colour moiré image of the test object with concave and convex surfaces (a). Line profile graphics of colour moir fringes in moiré pattern (b), modifed by two colour gratings with red and blue bars, the order of colour rulings is opposite ot two grids. The graphs illustrate the red green blue components of the data that was obtained by sampling along a straight line cutting through the moiré fringes. DAC, digital-to-analog converter, which is the measurement unit of the colour coordinates, that can take any integer value between 0 and 255 . 